\title{
13 The solo cross-national researcher of long-term care systems for older people
}

\author{
Henglien Lisa Chen
}

\begin{abstract}
The main aim of this chapter is to fill a significant gap in the discussion of qualitative research methods and methodologies in cross-national comparisons, illustrated by the example of a three-nation study conducted by a solo researcher exploring the long-term care of the elderly. The study aims to shed light on several key issues - time and space, comparability, culture and language - involved in the practical implementation of cross-national qualitative research. Cross-national qualitative comparison is highly demanding and requires language skills, cultural understanding, resources and time to produce a rigorous comparative instrument and outcome. Key approaches that address challenges faced by solo researchers are careful attention to geographic location and flexible timetable and programs for the collection of data; use of multidisciplinary knowledge to address the complexity of the research topic; application of a multi-method and multi-layer approach in data collection and analysis; identification of the caring culture; and sensitivity to national as well as local language. The chapter concludes with an analysis of the added value provided by the activity of the solo researcher activity in cross-national qualitative research.
\end{abstract}

\section{Introduction}

Comparisons of long-term care provision for the elderly have been carried out since the 1990s. This testifies to a growing interest in gaining a salient international perspective on policy and practice in the field of care for elderly people and in increasing opportunities for learning about it. Such interest derives from welfare systems having to face the ageing of populations and looking abroad for evidence regarding effective policies and practical implementations. In conducting cross-national research on long-term care of the elderly, many researchers have relied on existing literature or secondary quantitative data to explore the provision and cost of care (e.g., Glendinning, 1998; Österle, 2001). Only a small number of countries were studied qualitatively, by a few researchers equipped with intimate knowledge of the countries under study, so that a concrete, welldefined issue could be investigated in two or more national contexts (e.g., Jani-Le Bris, 1993; Ungerson, 2004). Such scarcity is not surprising because this methodological approach presents serious challenges and requires the researcher to be able to interpret information across demographics and social issues, political and cultural differences, economic constraints, and the scope of welfare services. An overview of the extensive literature from the last three decades shows that, in general, cross-national qualitative research is rarely conducted by a solo researcher, with the exception of Zulauf's (1999) study on migration. Rather, it has been usually undertaken collaboratively by research 
teams based in the countries in question, contributing their substantial insider knowledge of the country in which they live. Intimate knowledge of the country by the researcher is vital for a qualitative cross-national study to be carried out successfully. Yet, the present chapter argues that cross-national qualitative research conducted by a solo researcher who has substantial knowledge of the studied countries may at the same time provide an insider and an outsider view that creates a different perspective. The main aim of the chapter is, therefore, to fill a significant gap in the discussion of research methods and methodologies in cross-national comparisons, by describing a three-nation study of long-term care for the elderly, conducted by a solo researcher. I am a native of Taiwan who has become a practitioner and researcher in England, with an additional family connection to the Netherlands. Such insider/outsider status not only provides me with the opportunity to investigate different realities, but also facilitates consideration of the researcher's distinctive and interactive roles in the process of truth seeking (Merton, 1972). Moreover, as cross-national comparison studies tend not to provide sufficient information regarding their methods, this chapter explores methods and methodologies in qualitative cross-national research conducted by a solo researcher. It reflects on the central challenges of time and space, comparability, as well as culture and language encountered in the research process, to illustrate this distinctive methodological approach to qualitative cross-national research. The discussion that follows draws on a cross-national study I completed for my PhD degree, in 2008. To my knowledge, it is still one of the few examples of cross-national comparisons conducted by a solo researcher. The objective of the comparison was to investigate the policy and practice of long-term care for elderly people in three countries.

\section{Introducing the research project}

The research, "Successful Ageing in Long-term Care", was a 79-month, part-time PhD project that included conceptual, empirical and statistical data regarding the long-term care of the elderly in England, the Netherlands and Taiwan. The three industrial countries studied have faced increasing demand for long-term care. In England (following the National Assistance Act 1948, the NHS and Community Care Act 1990 and Care Act 2014) and the Netherlands (following the AWBZ 1968, the WMO 2007 and the long-term care reform of 2015), long-term care of older people has been embedded in state welfare over a long period of time. By contrast, economic welfare in Taiwan has only recently caught up with the European standard, and only as of late, since the 1990s, did the state start to invest more in care for older people. Unlike England and the Netherlands, which have a tradition of strong social protection by the state, Taiwan placed the emphasis on family- and community-based care, as well as on increased numbers of workers and professionals employed in the care sectors (Walker and Wong, 2005). Each country presents a different type of welfare regime, as classified by Esping-Andersen (1990). The core of Esping-Anderson's analysis is based on the notion of "decommodification", which is an indication of good welfare provision. Decommodification "occurs when a service is rendered as a matter of right, when a person can maintain a livelihood without reliance on the market" (Esping-Andersen, 1990, p. 3). The author defines three regimes that can be summarized as follows: (a) a social-democratic system that provides universal welfare and is characterized by a strong decommodification and redistribution element; (b) a conservative/ corporatist welfare system that supports the idea of class and status differentials, with 
minimal redistribution, where the benefit and welfare provisions are differentiated based on status; and (c) a liberal model that encourages a strong market-oriented welfare system for the middle and upper classes, has minimal decommodification, and provides a residual safety net for the poor. Of the three countries covered by the present study, England is liberal, encouraging a strong market-oriented welfare system, minimal decommodification and providing a residual safety net for the poor; the Netherlands is social-democratic, with universal state welfare provision, characterized by strong decommodification and retribution elements; and Taiwan is similar to Japan, in that it has a conservative familialist welfare regime, which supports the idea of class and status differentials, with minimal redistribution.

One principal aim of policies dealing with the ageing population in the three counties is to maintain elderly people in their own home for as long as possible. Nevertheless, both England and the Netherlands have found it difficult to sustain increases in state provision because of the significant economic effect of the necessary financing, whereas the Taiwanese government has found it necessary to provide expanded resources for its ageing population. Thus, the gap in responsibility between the state, individual and family is closing between the three countries. These similarities and differences mean that there is a potential for learning through cross-national comparative research (Doling, Finer and Maltby, 2005). Furthermore, by including Taiwan, the study filled an important gap in the comparison between Eastern and Western systems.

The study focused on exploring the quality of life of the elderly and the care they receive. I sought to understand how the systems supported their elderly and on what this capacity of each care system was based. Were some societies able to meet the needs of older people better than others? What were the successes and difficulties of each system in achieving better quality of long-term care? How could countries learn from one another in their search for solutions? The study provided an opportunity to broadly assess the three clearly different welfare systems, pointing out the complexity of comparison between countries with different funding issues, care market and care service patterns. I attempted to address the research questions by exploring the views and experiences of older people, extending the scope of the research to their families, care workers, care professionals, local administrators, service providers, civil servants and volunteer agency officials who had held care provision responsibilities. To minimize variation in the comparative research, the service users included in the sample were female, aged over 60, from the majority ethnic group and receiving formal care support. I chose to focus on women to reflect the demographic trend in the countries studied, because of women's more extended longevity compared to men's, and therefore, higher likelihood of them needing long-term care. To cover the widest possible range of provided services, in each country, five participants lived in their own home, two lived in nursing homes - and in England and Taiwan - two lived in residential care homes. Additionally, three (including one resident in the care hotel) lived in residential care type homes in the Netherlands. Of a total of 143 interviewees (48, 43 and 52 in England, the Netherlands and Taiwan, respectively), 28 elderly people were included in this study as shown in Table 13.1.

In the analysis that follows, the methodological challenges of space and time, comparability, culture and language are explored with reference to the position of the solo researcher. 
Table 13.1 Numbers of interviewees in present research

\begin{tabular}{lcccc}
\hline Interviewees & \multicolumn{4}{l}{ Number of interviews } \\
\cline { 2 - 5 } & England & Netherlands & Taiwan & Total \\
\hline Care recipients & 9 & 10 & 9 & 28 \\
Informal carers & 6 & 1 & 3 & 10 \\
Formal carers & 5 & 9 & 9 & 23 \\
Assessors & 11 & 8 & 11 & 30 \\
Service providers & 10 & 7 & 8 & 25 \\
Local administrators & 4 & 4 & 6 & 14 \\
National civil servants and NGOs & 3 & 4 & 52 & 143 \\
Total & 48 & 43 & & \\
\hline
\end{tabular}

\section{Space and time}

The geographic location of a study conducted in different countries, areas or cultures relevant to the aims of the research is important for both theoretical and methodological reasons (Tester, 1999). Research officially sponsored by countries is motivated primarily by the desire to promote and improve their own system. Often, effort has been limited by a methodology that focused on the most similar countries. Yet, comparing countries that are different from each other could also be informative, and learning social policy and practice from other countries is consistent with the phenomenon of globalization. Therefore, examining whether different welfare regimes - social democratic, conservative and liberal deliver a different quality of care, as well as the questions of why and how the particular issues of quality of life in old age are more prominent in one country than in other are the main focus of this study.

It was important to insure that at the micro-level, the localities in each country were not in too dissimilar geographic areas, to provide relatively comparable research material. Therefore, both service users and providers in the sample were selected from midland districts, suburban localities at the edge of medium-sized cities. Suburban locations were chosen because cities may have other problems obfuscating the picture, and rural areas are not typical of the industrial countries studied. A further reason for excluding urban and rural areas was that the former would arguably attract more, and the latter fewer, formal and informal resources. The experiences of elderly people in these areas could be too extreme and not represent the situation in the country. I also excluded the areas where I used to live or work, to avoid a possible conflict of interest.

An adequate time span is crucial to allow for the comparative analysis of policy implementation and the outcomes for users and carers over a period of time, both the time frame of the empirical research itself and the time frame of provision of the researched service (Tester, 1999). My research design focused on service recipients who had been receiving care for over three months, because the three-month period is adopted by UK practitioner guidelines for reviews of social care provision (Department of Health, 2002). Therefore at least a three-month experience of care is necessary for an elderly individual to form an opinion about the care they are receiving. In addition, field work was carried out within a comparable time frame (roughly three months of the same year in each country), to capture the practice in the three countries at proximately the same points in time. All three countries have implemented important reforms regarding the long-term care of elderly people. 


\section{Henglien L. C.}

The policy changes created some tensions in practice, which were particularly strong in the Netherlands and England. This was one of the reasons that the fieldwork was difficult to time. In the Netherlands, it took four attempts to establish contact with the regional assessment offices, as such attempts were immediately turned down. In England, my application to carry out the fieldwork in one local authority in the Midlands took four months of negotiation, and eventually was turned down. The successful application with another local authority took two-and-a-half months to obtain approval, because of the complexity of research governance procedures. The extensive time required for obtaining research approval in England, in comparison with other countries, is a result of English research governance procedures, and has also been reported by Zulauf (1999). Thus, the sequencing of the research in time and space for a solo researcher working in a complex environment is a key challenge; it requires paying attention to the local research culture, and careful planning, to be able to start preparations for fieldwork as early as possible.

Many research projects have to meet a strict timeline. Care policy and practice, as well as the lengthy research approval process mentioned above resulted in an ever-present challenge of racing against time (Delva and Altman, 2010). Solo researchers face additional difficulties, given the extensive literature and secondary data research in the target countries that they must absorb, which at the time were undergoing various reforms in care for the elderly. Research design for every country needs to be comparable with the design of research for other countries, and it needs to be ready and operational as soon as possible. To increase communication efficiency, interviewees in every country need to be contacted electronically before traveling to the country. Thus, in planning a cross-national project, a solo researcher needs to take into account these aspects and be able to work using a "hurry up and wait" (Delva and Altman, 2001, p. 605) approach. Time slippage in one part of the study means reprioritizing the research tasks, as there is always work to be done elsewhere. One such example is to arrange interviews with the civil servants and senior officials in national non-governmental organizations (NGOs). My initial attempts to do so in England were unsuccessful, at the time when the rest of the interviews in this country had been completed. I decided to conduct fieldwork in the other two countries, while making further attempts to recruit the interviewees at the national level in England.

\section{Comparability}

The second and most common methodological challenge that arises in cross-national research, which may affect the reliability of research findings, is how to insure comparability. The challenges are considerable, given variations between political systems, social policies, institutional frameworks and underlining cultures in different countries (Clasen, 1999). Increasingly, comparative studies argue for combining quantitative and qualitative approaches, to detect various differences between the countries being studied (Bennett and Elman, 2006). The present study involved two key stages. The first stage consisted of discussing and analyzing quantitative data from public sources (i.e., the World Health Organization (WHO), the Organisation for Economic Co-operation and Development (OECD), Eurostat and national governments) and from the research literature, to assess the similarities and differences between demographic and social issues, the cultural and political differences shaping policy objectives, economic constraints and long-term care services (for further discussion, see Chen, 2010). The second stage utilized qualitative methods to facilitate a more detailed examination of data collected from statistical and literature reviews. The present section focuses primarily on the qualitative phases of the research. 


\section{The conceptual framework}

Comparative analysis of long-term care may use the welfare system typologies, such as those proposed by Esping-Andersen (1990) and Abrahamson (1992), based mainly on social protection systems. The models and frameworks of Alber (1995) and of Attoneon and Sipilä (1996) further explore social care, which is an important constituent of long-term care in a comparative context. The various existing models and approaches contribute to our understanding of similarities and differences between welfare systems, but there is a need to build a conceptual framework specifically applicable to the study of long-term care for the elderly. Active Ageing (WHO, 2002) and Advancing Health and Well-Being into Old Age (IFSW, 2012) emphasize the importance of providing humanitarian assistance and adequate resources for care service programs, to insure that people are able to age with security and dignity, and continue to participate in community life. It is particularly important for those who are vulnerable and require support to achieve a good quality of life. The long-term care of elderly people is an extremely diverse concept, worthy of an in-depth and holistic look at the care systems being studied. The approaches underpinning this study, therefore, were based on multidisciplinary knowledge, derived from social policy (Doyal and Gough, 1991), the psychological concept of needs (Maslow, 1943) and the gerontological concept of successful ageing (Havighurst, 1961; Rowe and Kahn, 1997). Two aspects of a framework emerged: a theoretical one, derived from gerontology and psychology, suggesting that there are at least three components (care needs, social inclusion, and power and autonomy) that must be addressed for successful ageing to take place; and once concerning policy development and service delivery, certain elements of care delivery (such as partnership and resources) that need to be present if successful ageing is to be promoted.

\section{Interview design}

One of the cultural considerations in this project is the issue of power status of various interviewee groups within a country. Sohng (1994) has argued that many traditional empirical research methods are inherently biased in favor of the dominant social groups and fail to take into account the unique perspectives of vulnerable groups. Noticeably, within the field of health and social care, both practitioner and user knowledge have traditionally been marginalized (Cheston, Bender and Byatt, 2000; Beresford, 2003). This is one of the reasons why efforts have been made also to capture the perspective of diverse groups of respondents (i.e., care recipients and their relevant formal/informal carers and care professionals) on issues related to the quality of long-term care, and derived from the conceptual framework. To improve the balance of interests in each interviewed group, when framing the questions for the interview questionnaires, the valuation of different types of knowledge that different interviewee groups might contribute was taken into account. Several scholars (e.g., Lewis, 2001; Marsh et al., 2005) have provided an overview of the types of knowledge that should inform social care and practice:

- Organizational knowledge of governance and policies.

- Practitioner knowledge: personal, practice, and context-specific.

- User knowledge, with first-hand living experience and reflection on care.

- Research knowledge - the most "plausible" source but requiring a "broad church" interpretation.

- Community policy, with social and political drivers determining the issues of significance. 
Such approaches offer no implied hierarchy of evidence, but rather, different types of knowledge are seen as suitable for differing purposes. As a result, six interview schedules were used in the fieldwork:

- The interviews with service recipients focused on how much they were involved with decisions about their care, what effect the services had on their lives, how they thought care services were meeting their needs and what should be included in the provision of long-term care services.

- To evaluate the contribution of informal and formal caregivers to the care system and to examine the process of long-term care from their perspective, two separate interview schedules were designed. The interviews were focused on how care affected the caregivers' individual welfare in the family and in society, how it may affect them in the future, what they considered to be important to them as caregivers, how much they had been involved with the assessment of the elderly individuals for whom they cared and what they thought was important for the long-term care of elderly people.

- Interviews with assessors explored how they put policy into practice, how eligibility criteria and the decision of the assessment system affected the care support the elderly received, how assessors worked in partnership with others, what they thought was the greatest challenge faced by those who provided elderly clients with long-term care and what could be done to insure these challenges were met.

- The aim of the interviews with service providers was to examine the process of care provision and management quality, to find out the state of their current staffing and financial situation, to find out their principal concerns and the quality of their relationship with service recipients, various professionals and other agencies.

- The interviews at the county level aimed to determine how the local authority implemented national policy, how the local authority insured that long-term care met local needs and how it met national targets, what the local authority thought were the important issues that needed to be addressed to improve long-term care for the elderly and what the local authority thought were the important issues that needed to be addressed by national government.

- The interviews with participants at the national level examined how long-term care policy was formed and implemented, how policy set standards of care and what the national influence on local practice was.

Although it may be argued that to fully represent the expertise of each interviewee group is one of the ways to improve participant inclusion, no research can claim to be 100 percent non-biased. Some of the complexities and challenges of comparative research that came into play derived from the cultural variation in gaining access to interviewee groups and in their responses, discussed below.

\section{Choice of methodology for coding and analysis}

Even an empirically rich comparative framework may produce poor findings if it is methodologically inconsistent and its objectives are unclear. To maximize the comparative reliability in the research findings across the countries studied, many crossnational researchers use multilingual teams applying multi-layer analysis to carry out the analytical work. For example, a European Union (EU) housing study (Quilgars et al., 
2009) focused specifically on the analysis of the individual countries studied by each team from the country of origin; subsequent comparative analysis was carried out by the leading research team. Nevertheless, the differences between countries in disciplinary and interdisciplinary approaches to analysis may result in differences in the application of the findings (Tester, 1999). Some relevant data may be filtered out or interpreted differently by researchers in an individual country before integrative analysis, which may compromise the validity of comparison. By contrast, a multi-layer analysis by one researcher in a cross-national analytical process arguably makes the methodological approach more consistent.

Thus, two layers of comparative analysis were employed in the present study. The first focuses on each interviewee group across the countries studied, then analyzes the themes shared by all the groups in the three countries. This layer provided fewer samples to consider at each step, allowing careful scrutiny of data at the first stage of the overall analysis, and preventing issues from being overlooked, as they might be when only a single layer is considered. In addition, multiple organizing approaches in the analysis of each layer were adopted by using Microsoft Excel to display relevant excerpts selected systematically, and by applying Becker's "sequential" methodology in the systematic coding and analysis of the interviews (Becker and Bryman, 2004), revealing certain thematic characteristics. Miles and Huberman (1994) have shown that table structures are powerful tools for data analysis. The advantage of using a template or code manual is that they may be more focused and time efficient than other organizing styles, such as editing and immersion/crystallization. Its disadvantage, however, is that when used alone, there is a potential for information to be overlooked (Crabtree and Miller, 1992). Displaying data in the maps and matrices (Dey, 1993) by using MindMan software at the third stage was another powerful means of discovering the relationships between categories, thus providing a visible way to explore possible links and associations between code-sorted segments of data. The software was used alongside Excel to reinforce and confirm the logical links between participants' views within and across the countries studied.

For a solo researcher, studying another long-term care system inevitably requires making some comparison with one's own or others with which one is familiar. To justify the findings, comparability in cross-national research is crucially important. In addition, certain supplementary mechanisms need to be put in place to prevent bias resulting from work in isolation. The present study sought to minimize bias by basing its conceptual framework on multidisciplinary theories, by inclusion of different types of knowledge that various interviewee groups were expected to contribute, and by adopting multimethodology and multi-layer analysis. It is reasonable to question whether, given the researcher's potential biases, the multi-layer and multi-methodology analysis conducted by a situated solo researcher might nevertheless be flawed. Note, however, that a solo researcher project is not carried out in isolation. Proactive seeking of the opinions of other scholars, care recipients and care providers can significantly confirm or challenge the analysis performed by a solo researcher. In my research, first, I shared the early findings with all participants for feedback. Second, the research itself was evaluated annually by two university professors who were not supervising the project. Third, the ongoing research findings were presented and shared with scholars, elderly people and caregivers from the countries studied at nine international conferences and congresses. All confirmations and criticism were incorporated into the findings, providing further justification and affecting the overall analysis. 


\section{Culture}

Cross-national comparisons benefit from a full appreciation of culture, such as language, values and attitudes (Van de Vijver and Leung, 1997). For the present study, an additional aspect of culture required special recognition: the culture of caring, including caring values, attitudes, systems and institutions. For this reason, single-person, cross-national studies that attempt to cover the full effect of policy implementation may be difficult to undertake, although, in this case, the researcher can claim to have cultural knowledge and experience, having lived in all three countries.

\section{Gaining access: organic approach and snowball method}

Recruiting participants was difficult and required a great deal of effort. As noted, the principle of the organic approach in this project presumed that interviewees who were the relevant care actors would have either a direct or indirect caring relationship with the included service recipients. One of the most practical methods of securing interviewees is through snowball sampling, which involves the personal recommendation of a contact (Arbert, 1993). Differences between the structure and operation of the care systems in each country, however, meant that participants could not be recruited in an identical fashion, but appropriate adjustments had to be made.

Taiwan, with its conservative welfare regime, places greater emphasis on private provision, therefore not all elderly service recipients are known to the government. Service providers, therefore, had to be involved in the process of recruitment. By contrast, in the social-democratic Netherlands, the vast majority of elderly service recipients are known to the government. At the same time, in that country there is a greater emphasis on privacy and rights of individuals, making a direct approach more difficult; therefore, an appeal had to be made to the general public in the community, by handing out leaflets inviting service recipients to contact the researcher. In England, social services identified the elderly who met the research criteria; assessors discussed the study with individuals who were potential interviewees, and passed details of those who had given consent to the researcher.

Differences in the care system also required different approaches to gain the cooperation of relevant authorities in each country. In England, where the local authority has the main responsibility for meeting the needs of care recipients, the research recruitment began with approaching local authority social services with responsibility for the care of older people. It then became possible to seek consent from participants at the local and national levels (center-to-bottom and top approach). In the Netherlands, with its pillar-like social structure and data protection regulations, many consultations took place between the researcher and various groups (bottom-to-top). In Taiwan, which has a strong hierarchical social structure, the strategy of obtaining approval at ministerial level was sufficient, and everything else then fell into place (top-to-bottom).

One limitation of the snowball method has to do with the power relations within the care system. In England and Taiwan, these can become more problematic in situations of a top/ center-to-bottom approach. Some respondents may not turn down the interview because someone who has higher authority referred the researcher to them, and thus they do not wish to disappoint that person. The bottom-to-top approach in the Netherlands may have meant that recruited participants were more likely to be autonomous, and the elderly people volunteered without any pressure from other actors. Another potential limitation and difficulty of the snowball method is that it includes only individuals within a specific network 
of people, which may introduce a bias into the research findings (Arbert, 1993). In the case of this study, differences in the culture of caring affected the personal recommendations of contact and the final participant sample. There is little doubt that informal caregivers played a significant role in the care of their older relatives. Elderly individuals' perception of informal caregivers in the three countries, however, resulted in varying involvement of caregivers in the research. Access to informal caregivers was not always possible in England and the Netherlands because some English and Dutch elderly individuals saw themselves as being independent while receiving formal support, and therefore as not being dependent on their families. In Taiwan, some elderly people considered the research involvement as a burden on their family or informal caregiver, many of whom were working full time.

The organic approach to the recruitment and selection of interviewees from every level of the care system, to represent the care system as a whole, can be complex and difficult, especially in cross-national research. To meet the criteria, a complete cluster had to include a minimum of one service recipient, a formal or an informal caregiver, an assessor and a service provider. Although there was close cooperation with potential participants, some of the care recipients or their relevant caregivers refused to participate. Two elderly care recipients in England were too frail to be interviewed. In Taiwan, three care recipients were too frail to participate, and one assessor and one formal caregiver could not be contacted. The care recipients who were too frail to participate were either suffering from dementia or unable to communicate with the researcher for other reasons (e.g., one English service recipient was too distressed by having to sell her home). In the Netherlands, one care recipient who had given consent did not meet the research criteria, and four assessors could not be contacted. This was discovered at a later stage, and, as a result, 11 clusters could not be completed and 36 interviews had to be abandoned.

To represent the different structures of care systems and different individual needs of elderly people who received different levels of support in the three countries, the number of participants in each cluster could not be identical. The number of participants at the national level and the local administrators in the study (Table 13.1) reflected the level of public responsibility characteristic of the English care system, with fewer departments and NGOs than in the other two countries. In England and Taiwan, most of the service recipients had a regular, main, formal caregiver. This was not quite as often the case in the Netherlands, however. Although the original principle of interviewee selection was still maintained, it worked out a little differently in the Netherlands. When there was no one main caregiver, one was selected at random. The culture and political differences identified in this section suggest the complexities in identical versus equivalent comparison approaches to recruiting participants.

\section{Interviewees' responses}

One of the general difficulties of involving elderly people who receive long-term care in research is that they are likely to have experienced the effects of the loss of health, family and independence. In the case of formal/informal caregivers and assessors who provide front-line care, it was recognized that they too were in a vulnerable position. In many instances, they displayed some stress stemming from the demanding responsibilities of their role. It was important to allow time for interviewees to express their feelings and to insure that individual interviewees realized they were free to decline to take part or answer only certain questions, without any negative consequences. 
As a result of seeking to take Eastern and Western cultural and political contexts into consideration, there was some variation in the responses. For example, the idea of a consent form worked well in England, but not in the Netherlands and Taiwan, because of different views of how agreement to participate in research should be expressed and presented. In the Netherlands, nearly all the interviewees saw their verbal agreement to participate in this research as equivalent to the signing of a consent form. In Taiwan, nearly all of the interviewees at local and municipal levels did not welcome, indeed were frightened by the offer of, a consent form. They saw the consent "form" as an official constraint on their voluntary contribution toward the research, and did not like to think of it as part of a government investigation. This difficulty was reduced by providing explanations and examples to the interviewees when required. The physical condition of the elderly (hearing, vision and cognition) affected the interviews to some degree, but this was minimized by the researcher's own experiences with older people as a social worker. Interviews lasted for an hour on average, with the exception of the interviews with the elderly. English and Dutch service recipients were expected to be less at ease because they were being interviewed by a foreigner. Yet, the length of the interviews suggests that Taiwanese elderly respondents were, in general, less forthcoming and more private than those in England and the Netherlands (approximately 30, 50 and 40 minutes, respectively).

\section{Visual method}

Whenever relevant for the study, photographs were taken to provide an additional perspective on the experience of care. Several anthropologists have stated that cultural products, such as visual materials, reflect social reality in various ways, and are among the best approaches to the illustration and elaboration of research findings (Albrecht, 1954; Alexander, 2008), especially when presenting cross-national, long-term care research to readers who are not always familiar with the countries studied. Visual methods, however, are still relatively uncommon in cross-national social policy and practice research because most research of this type has been conducted by social policy, health and social care scholars.

Cross-cultural studies, such as those of Dingwall, Tanaka and Minamikata (1991, cited in Chaplin, 1994, p. 195), which used visual images to compare parenthood in the UK and Japan, have argued that a comparison of images can be an effective general indicator of cultural differences, because images show a wealth of detail about the social situation they depict. The present study employed the strength of the visual image, which is information-rich, to draw attention to what is real and to contribute to the understanding of the everyday life of care by giving a more immediate and detailed account of people than is possible by a verbal description (Chaplin, 2002). In the present study, I used photographing as a visual recording method during the field research. The visual inventory was carefully classified and assisted me in reflecting on the views of the participants, especially of those who are disempowered by society. Nonetheless, visual materials, despite their potential, require that careful attention be paid to their interpretation. First, they are liable to create a problem of ambiguity, as different people may interpret them in different ways (Alexander, 2008). Second, photos can capture only a brief moment in time and a fraction of space. Third, consent needs to be obtained from the participants to be photographed, but if they are aware that a photo is being taken, it may affect their natural behavior. 


\section{Language}

Last but not least are challenges related to the multi-linguistic competence of a solo researcher. Language can present a key obstacle to effective international comparison, because it is not merely a medium for communication, but part of a wider system reflecting institutions, thought processes, values and ideology. This could mean that the approach to a topic and its interpretations may differ according to the language of expression. Comparative analysis is rarely able to achieve exact linguistic equivalence in concepts. Definitions acceptable in one country may not be understood elsewhere. The aim must be to devise functional equivalents, which need further explanation for use in a comparative context (Tester, 1999). For instance, the term assessor refers to all the people who formally assess elderly individuals' needs for formal care, covering a range of occupations that can vary a great deal in the countries studied. These can include social workers employed by the local authority, nurses and occupational therapists in England; social workers, physiotherapists, nurses in a regional assessment office, and nurses and social workers from service providers in the Netherlands; nurses contracted by a local authority, and nurses, social workers, physiotherapists and nutritionists from service providers in Taiwan. Hence, the terminology used in each country and by each interviewee group became important and had to be considered carefully.

In a world of social science research where good language skills are a rare commodity (Ungerson, 1996), a much more common strategy is to put together research teams made of native speakers from the target countries. For an international project by a solo researcher to be successful, a certain level of linguistic and cultural affinity of the researcher with the respondents is essential (e.g., in the national languages and local dialects), in addition to assistance from professional interpreters and translators. It helps facilitate good working relationships with the participants and minimizes translation problems at the critical stage of data collection and transcription.

In the present study, several measures were adopted at the stage of formulating research questions to overcome the language barriers. First, regular visits to the target countries, related to the research topics, as well as reading the relevant literature in the language of origin, helped me gain a greater understanding of the variations in terminology used in each country. Second, the interviews covered the same questions translated into each of the languages used in the study, following a pilot study (conducted with 21 interviewees) in the three countries, with some ensuing adjustments made to insure that the interview questions were clear and covered comparable areas of concern in the three countries.

The process of interviewing further confirmed the credibility of the use of the language of origin. For example, although many of the Dutch interviewees were fluent speakers of English and the use of English could have greatly increased the accessibility of their views, many of them spoke in their first language when responding to nearly all the interview questions to address sensitive care-related issues. What added to the complexity of conducting the interviews, especially with the elderly in the Netherlands and Taiwan, was that local dialects and provincial languages were frequently used. As a solution in this study, when a problem of communication with Dutch elderly individuals arose, was to avail myself of additional support from a colleague. To prevent the problem of mistranslation and misinterpretation, verbatim interview transcripts in both the original language and English were performed by the solo researcher and professional transcribers in each country, to minimize the possible loss of nuance and culturally determined meaning (Ungerson, 1996). 


\section{Conclusion and implications}

This chapter detailed the challenges and opportunities in conducting cross-national research in long-term care of older people by a solo researcher using qualitative methods, which has been rarely been carried out or discussed. Qualitative cross-national comparisons have the potential to provide valuable analytical insights into a range of outstanding long-term care problems and to facilitate a deeper understanding of care policies and their implementation. This chapter provided support for such work by demonstrating the benefits and feasibility of conducting qualitative comparative research by a solo researcher, in view of the fact that cross-national studies are lacking because of the challenges in their practical implementation. It offers examples of facing the challenges in study design and data collection and analysis, based on the experience of solo cross-national research of the long-term care for elderly populations. As in any research, it is important that theory and data correlate, and that issues of validity, reliability and ethical considerations be addressed. The need for comparability is an additional aspect (Tester, 1999). A rigorous cross-national comparison research demands extensive language skills, cultural understanding, resources and time. To achieve the research aim, it was essential for the researcher to collect the information about the relative effects of different actions on actual welfare outcomes in different countries. Some of the difficulties could be addressed by understanding the contexts in which comparisons were made. Empirical research conducted by a solo researcher helped maximize the consistency of data collected cross-nationally. The determination of equivalence in the fieldwork was insured by great attention paid to careful and detailed planning, based on continuity, reflection and adaptability. A delicate balance was required between sensitivity to these complex issues and ensuring, at the same time, that the project moved along without falling behind on important comparability criteria. Multiple methods in the overall analysis helped obtain consistent and systematic results, and avoid methodological pitfalls. More research is needed on ethical issues concerning a broader range of vulnerable elderly people, such as those with multiple health problems, and those who are caregivers. The ethical approval process varies across the countries studied, and lead times are especially protracted in England; researchers should consider this when planning their research.

In conclusion, the solo research experience presented in this chapter indicates that the challenges of cross-national research carried out without national research teams in the target countries can be successfully addressed. Indeed, in some respects, a single researcher working cross-nationally may overcome some of the challenges in cross-national research more broadly, thus contributing added value to the research process.

\section{References}

Abrahamson, P. (1992). Welfare pluralism: towards a new consensus for a European social policy. In: L. Hantraiset (ed.), The mixed economy of welfare. Cross-National Research Paper No. 6, Loughborough University, Loughborough.

Alber, J. (1995). A framework for the comparative study of social services. Journal of European Social Policy, 5(2), 131-149.

Albrecht, M.C. (1954). The relationship between literature and society. American Journal of Sociology, 59(5), 425-436.

Alexander, V.D. (2008). Analyzing visual materials. In: N. Gilbert (ed.), Research social life. London: Sage.

Arbert, S. (1993). The research process. In: N. Gilbert (ed.), Research social life. London: Sage.

Attoneon, A. and Sipilä, J. (1996). European social care services: is it possible to identify models? Journal of European Social Policy, 6(2), 87-100. 
Becker, S. and Bryman, A. (eds) (2004). Understanding research for social policy and practice: themes, methods and approaches. Bristol, UK: Policy Press.

Bennett, A. and Elman, C. (2006). Qualitative research: recent developments in case study methods. Annual Review of Political Science, 9, 445-476.

Beresford, P. (2003). It's our lives: a short theory of knowledge, distance and experience. London: Citizen Press.

Chaplin, E. (1994). Sociology and visual representation. London: Routledge.

Chaplin, E. (2002). Photographs in social research: the residents of South London Road. Everyday Cultures Working Papers, no. 2, Open University.

Chen, H.L. (2010). Cross-national inequalities: long-term care provision in the East and West. International Journal of Sociology and Social Policy, 30(3/4), 167-181.

Cheston, R., Bender, M. and Byatt, S. (2000). Involving people who have dementia in the evaluation of services: a review. Journal of Mental Health, 9(5), 471-479.

Clasen, J. (ed.) (1999). Comparative social policy: concepts, theories and methods. Oxford: Blackwell.

Crabtree, B.F. and Miller, W.L. (1992). Doing qualitative research. Newbury Park, CA: Sage.

Delva, J. and Altman, M.C. (2010). International research. In: B. Thyer (ed.), The handbook of social work research methods. London: Sage.

Department of Health (2002). Fair access to care services: guidance on eligibility criteria for adult social care. Local Authority Circular, Department of Health, London.

Dey, I. (1993). Qualitative data analysis: a user-friendly guide for social scientists. London: Routledge.

Doling, J., Finer, C.J. and Maltby, T. (2005). Ageing matters: European policy lessons from the east. Aldershot: Ashgate.

Doyal, L. and Gough, I. (1991). A theory of human need. Basingstoke, UK: Macmillan.

Esping-Andersen, G. (1990). The three worlds of welfare. Cambridge, UK: Polity.

Glendinning, C. (ed.) (1998). Rights and realities: comparing new development in long-term care for older people. Bristol, UK: Policy Press.

Havighurst, R. (1961). Successful ageing. The Gerontologist, 1(1), 8-13.

International Federation of Social Workers (IFSW) (2012) Advancing health and well-being into old age. Available at: http://ifsw.org/policies/ageing-and-older-adults/ [Accessed 30 August 2019].

Jani-Le Bris, H. (1993). Family care of dependent older people in the European community, European foundation for the improvement of living and working conditions. Office for Official Publications of the European Communities, Luxembourg.

Lewis, J. (2001). What works in community care? Managing Community Care, 9(1), 3-6.

Marsh, P., Fisher, M., Mathers, N. and Fish, S. (2005). Developing the evidence base for social work and social care practice: using knowledge in social care report. Social Care Institute for Excellence, London.

Maslow, A. (1943). A theory of human motivation. Psychological Review, 50(4), 370-396.

Merton, R. (1972). Insiders and outsiders: a chapter in the sociology knowledge. American Journal of Sociology, 78(1), 69-103.

Miles, M.B. and Huberman, A.M. (1994). Qualitative data analysis: an expanded sourcebook. 2nd ed. Thousand Oaks, CA: Sage.

Österle, A. (2001). Equality choices and long-term care policies in Europe: allocating resources and burden in Austria, Italy, the Netherlands and the United Kingdom. Aldershot: Ashgate.

Quilgars, D., Elsinga, M., Jones, A., Toussaint, J., Ruonavaara, H. and Naumanen, P. (2009). Inside qualitative, cross-national research: making methods transparent in a EU housing study. International Journal of Social Research Methodology, 12(1), 19-31.

Rowe, J.W. and Kahn, R. L. (1997). Successful ageing. The Gerontologist, 37(4), 433-440.

Sohng, S.S. (1994). Are traditional empirical research methods inherently biased against people of colour? Yes. In: W. Hudson and P.S. Nurius (eds), Controversial issues in social work research. Boston, MA: Allyn \& Bacon. 
184 Henglien L. C.

Tester, S. (1999). Comparative approaches to long-term care for adults. In: J. Clasen (ed.), Comparative social policy: concepts, theories and methods. Oxford: Blackwell.

Ungerson, C. (1996). Qualitative research methods. In: L. Hantrais and S. Mangen (eds), Crossnational research methods in the social sciences. London: Pinter.

Ungerson, C. (2004). Whose empowerment and independence? A cross-national perspective on "cash for care" schemes. Ageing \& Society, 24, 189-212.

Van de Vijver, F. and Leung, K. (1997). Methods and data analysis for cross-cultural research. Thousand Oaks, CA: Sage.

Walker, A. and Wong, C.K. (2005). East Asian welfare regimes in transition: from Confucianism to globalisation. Bristol, UK: Policy Press.

World Health Organization (WHO) (2002). Active ageing: a policy framework. Geneva: WHO.

Zulauf, M. (1999) Cross-national qualitative research: accommodating ideals and reality. International Journal of Social Research Methodology, 2(2), 159-169. 